\title{
Research on Modular Design Method of Mechanical Products
}

\author{
Zheng Kai \\ Xi'an Transportation Engineering College, Xi'an, Shaanxi, 710300, China
}

Keywords: Modular design, Mechanical products, Personalized

\begin{abstract}
Modular design is an advanced design method. Its core idea is to divide the system into several modules according to their functions. Through different combinations of modules, products of different varieties and specifications can be obtained. The overall planning of modular products is very important to the whole design system. If the design is blindly carried out without planning or the planning method is improper, the expected effect will not be achieved and it is difficult to respond quickly to changes in the market. Today's users need personalized and diversified products, and at the same time, they want the cost and price of products to be as low as possible. Modular design through the restructuring of product structure and design process to achieve the mass production of customized products and personalized mass production conditions at the cost of mass production. In mechanical product design, how to deal with module division and module combination is the key to the success of modular design.
\end{abstract}

\section{Introduction}

Today's market changes rapidly, and enterprises must constantly apply new technologies to quickly adapt to the changing market environment. Modular design is an advanced design method. Its core idea is to divide the system into several modules according to their functions. Through different combinations of modules, products of different varieties and specifications can be obtained [1]. Machine tool products with complex structures are especially suitable for adopting modular design, because modular design is beneficial to the material selection and management of products, to the disassembly of products, and to the recyclability of products [2]. Modular design is a design method that divides and designs a series of functional modules on the basis of functional analysis of products with different functions or the same functions, different performances and different specifications within a certain range. Through the selection and combination of modules, different products can be formed to meet the different needs of the market [3]. In the products with modular design structure, since the module is easy to be disassembled and assembled from the whole machine, the maintenance can be disassembled and assembled by taking the module as a unit and is not limited by tools, test equipment, operation space and the like, thus greatly improving the maintenance conditions and simplifying the maintenance work [4]. Nowadays, users need personalized and diversified products, and at the same time, they want product costs and prices to be as low as possible. Modular design realizes mass production of customized products and personalization under large-scale production conditions at the cost of mass production through reorganization of product structure and design process.

With the acceleration of changes in market demand and the development of advanced manufacturing technologies, modular design can shorten product development cycles and respond quickly to market changes, which is conducive to product maintenance, upgrades and reuse. Through modular design, the product is broken down according to different functions, and the design of each module and the reorganization between modules are used to realize the new design of the product, which makes up for the lack of case-based reasoning design for mechanical products [5]. Today's market is changing rapidly, and companies must continuously apply new technologies to quickly adapt to changing market conditions. The changing environment is attributed to a new generation of users who can purchase products globally. In the design of mechanical products, how to properly handle module division and module combination is the key to the success of modular design [6]. The traditional mass production method has caused enterprises to often launch new 
products every few years, and most products can only provide a limited number of functional features. In this case, the ability to quickly produce low-cost, high-quality products that satisfy customers is the key to a company's success. Modular design realizes mass production of customized products and personalization under mass production conditions at the cost of mass production through reorganization of product structure and design process, allowing enterprises to quickly form new types by improving certain parts of the product product.

\section{The Basic Idea of Modular Design}

Modularization of products refers to the creation and design of a series of functional modules on the basis of functional analysis of products with different functions or the same functions, different performances and different specifications within a certain range. Different products are formed through the selection and combination of modules to meet different market demands. The benefits of modular design are long-term, extensive and indirect, and the evaluation of modular benefits is difficult [7]. It is necessary to formulate a set of universal modular benefit evaluation system for mechanical product parts, so as to reduce repetitive work, improve the consistency of modular benefit evaluation for mechanical product parts, and promote and guide mechanical manufacturing enterprises to carry out modular design. There are many kinds of mechanical products, many parts and components, large structural changes, complex production process, long product life cycle, and need to carry out maintenance and recycling of parts and components. The modular design process of products generally includes module division, module creation and scheme design. Modular design of mechanical parts is difficult and requires a lot of work and investment. Therefore, it is necessary to carry out modular benefit evaluation of mechanical parts.

According to the form and degree of products covered by modular design in product series, people often divide modular design into horizontal series modular design, vertical series modular design, cross series modular design and full series modular design. Using the existing technology to establish a multi-mode collaborative work environment. Collaborative work support platform with integrated multimedia mode. The cooperative design and operation process is shown in Figure 1.

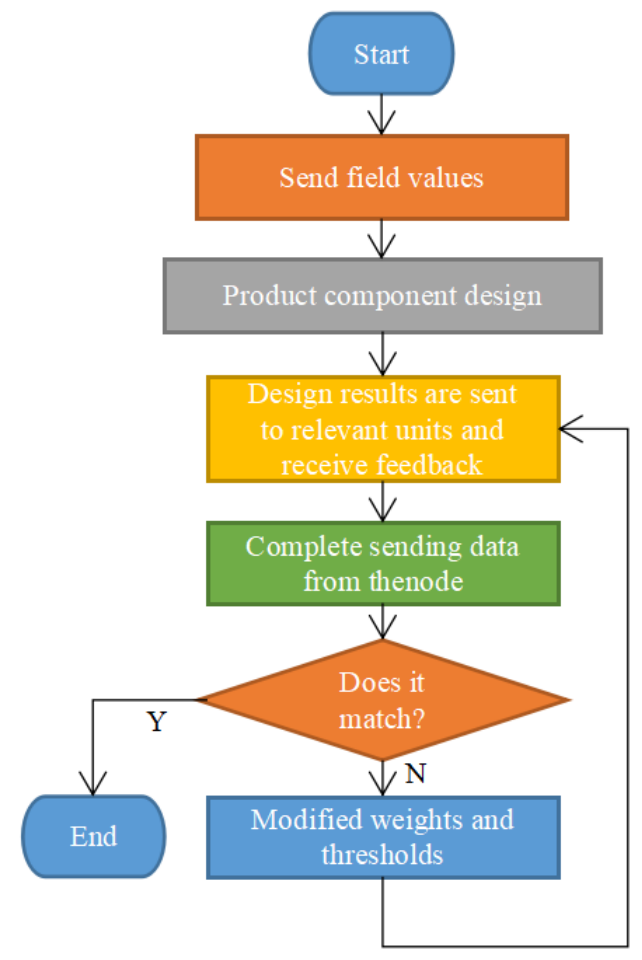

Fig.1 Cooperative Design Operation Process

The performance requirements of modular design are improved and met from four aspects: real size, economy, energy saving and coordination. The overall network density, center potential and 
number of nodes are analyzed as shown in Table 1.

Table 1 Network Structure Data

\begin{tabular}{|l|l|l|l|}
\hline Network & Number of nodes & Network density & Central potential \\
\hline Encounter information & 376 & 0.734 & 0.397 \\
\hline Mutual information & 398 & 0.842 & 0.432 \\
\hline Weighted summation & 352 & 0.875 & 0.418 \\
\hline
\end{tabular}

In modular design, the product must first be divided into several modules, and then the module is taken as the basic unit for design. In view of the difficulty in transporting large-scale equipment, it can be divided into modules for transportation. Modular products are mainly designed, produced and managed in units of modules. The division of modules is a very critical step in modular design. The common goal is to realize the combination of all products within the product range with the minimum number of modules. The reasonable division of modules has a great influence on the performance, appearance, universality and cost of products. Modular design decomposes mechanical products according to their functions, which is helpful to the implementation of CBR specific steps, while CBR technology is helpful to incorporate the design experience that is difficult to express into the module [8]. After determining the functions of different levels of the product, the product should be decomposed. In the physical decomposition of the product, the product is decomposed into basic physical elements, which can realize the function of the product after integration. The whole process of product modular design includes the establishment, control, acceptance and implementation of modular projects, and the evaluation of modular benefits needs to be carried out in each stage to ensure that modular design achieves better benefits. Module division can be divided into different methods and levels, but generally it is mainly analyzed and discussed from the perspective of function.

\section{Modular Integrated Design Method for Mechanical Products}

\subsection{Framework Model of Modular Integrated Design Method}

After determining that modular design can be carried out, the modularity degree of the existing products should be evaluated to determine how to carry out modular design on the products. After the work target is determined, the appropriate modular process should be selected for module design and development, and finally the effect of the modular results should be evaluated. In the product modular design system based on case reasoning, the parametric feature model of the case is established by applying feature modeling and parametric technology. On this basis, through the transfer-in of characteristic parameters, instance characteristic models with different parameter values are generated, thus establishing the instance library of the system [9]. At the present stage, the product is usually divided into several units on the basis of functional analysis so that these units become functional modules, and then the overall functions of the product are realized by the functional module system. Modularization generally refers to the planning, design and production organization of products or systems using the concept of modules. Modular design of products is to divide and design a series of modules based on functional structure analysis of products with different functions or the same functions, different performances and different specifications within a certain range. According to the modularized work target and the selected product or product family, the appropriate modularized process can be selected, thus increasing the flexibility and comparability of the integrated method.

\subsection{Characteristics of Integrated Methods}

After demonstrating the appropriate degree of modularity, it is necessary to analyze how the product is modularized. When the product has modularity higher than the average score, the product will accept modular design. The lower the level and level of the module, the simpler the module, the easier it is to improve the degree of generalization, but the more complex the management of product manufacturing and assembly. The shape features of mechanical parts mainly reflect the geometric shape information of the parts. It is the most basic feature of the parts and also the carrier 
of other features [10]. Therefore, feature decomposition mainly focuses on the decomposition of shape features. Enterprises can select some of these indexes for combination evaluation according to the product conditions, and can decompose them into granularity that can be quantified according to needs. According to the requirements of customization, necessary changes should be made to the modular structure of the modular strategy. The degree of modularity should not be confused with the suitability analysis of modularity. Retrieve the instance closest to the design requirements from the reducer instance library, and compare the design requirements with the attribute parameters of the most similar instance to find out the attribute parameters that do not meet the design requirements [11]. Modules should be as independent as possible, and there should be little or no functional crossing between modules. In this way, it is not only conducive to operation, assembly, disassembly, experiment and transportation, but also conducive to maintenance.

\section{Conclusions}

In today's market environment, the trend of product development is to speed up product upgrading and diversification of user demand. Modular design is an inevitable measure to meet the needs of diversified products, personalized products, small batches and short production and maintenance time. The background of evaluating the benefits of mechanical product modularization is: product modularization has good benefits, but product modularization requires investment and time cost, and not every product is suitable for modularization. Modular design of mechanical parts is difficult and requires a lot of work and investment. Therefore, it is necessary to carry out modular benefit evaluation of mechanical parts. Enterprises can select a number of indicators to carry out combined evaluation according to the product situation, and can be decomposed into granularity capable of quantitative calculation according to needs. The division of modules is a very critical step in modular design. The common goal is to realize the combination of all products within the product range with the minimum number of modules. The reasonable division of modules has a great influence on the performance, appearance, universality and cost of products. According to the requirements of customization, necessary changes should be made to the modular structure of the modular strategy.

\section{References}

[1] Song miaorei, Zhang Yanhong. Research on product modular collaborative rapid design technology based on Web3D [J]. Mechanical research and application, 2016, 29 (2): 195-198

[2] Ma Junlin, Sao, Zheng Liming. Modular multidimensional linear motion electromechanical platform [J]. Development and innovation of electromechanical products, 2015 (06): 48-49

[3] Xiao qinlan, Yang Qin, Li Yi, et al. Research on modular design method for product family [J]. Modern machinery, 2014 (2): 49-52

[4] Zhang Nan, Li Fei. Modular design of digital products in the context of Internet of things [J]. Mechanical design, 2014 (1): 126-128

[5] Yang Qin, Xiao qinlan, Peng min. modular design method research and application of product family [J]. Mechanical design and research, 2014, 30 (1): 5-7

[6] Liu Xianwei. Modular design method and its application in mechanical design [J]. Modern manufacturing technology and equipment, 2016 (6): 57-58

[7] Li Tingting, Wang Zongyan, Wu Shufang, et al. Application of parametric finite element analysis in modular products [J]. Mechanical design and manufacturing, 2016 (1): 115-118

[8] Huang Xiao, Wang JieQiu. Design innovation of multifunctional modular suitcase [J]. Mechanical design, 2014 (5): 120-122

[9] Zhou youhang, Zeng Lei, Zhang Qiao. Module division method based on product core parts [J]. 
Mechanical design and research, 2014 (2): 1-3

[10] Sheng Zhenxiang. Control strategy and design method for intelligent product development of wood-based panel machinery [J]. China wood based panel, 2016 (9): 1-5

[11] Gu Xiao. 3D modularization of non-standard equipment design and process [J]. Science and technology Shangpin, 2016 (6): 112-113 